STUDIA ROMANICA POSNANIENSIA

\begin{tabular}{lll}
\hline UAM & Vol. 41/3 & Poznań 2014
\end{tabular}

\author{
JEAN-MARIE LALLOUET
}

lallouet@amu.edu.pl

Université de Poznań

\title{
LES POSSIBLES APPLICATIONS DU FRANÇAIS SUR OBJEC- TIF UNIVERSITAIRE DANS UN DÉPARTEMENT DE LANGUES ROMANES
}

\begin{abstract}
Jean-Marie Lallouet, Les possibles applications du français sur objectif universitaire dans un département de langues romanes [Potential implementations of French for academic purposes in the Institute of Romance Philology], Studia Romanica Posnaniensia, Adam Mickiewicz University Press, Poznań, vol. XLI/3: 2014, pp. 63-72. ISBN 978-83-232-2759-5. ISSN 0137-2475. eISSN 2084-4158. DOI: 10.7169/strop2014.413.005
\end{abstract}

French for academic purpose is a recent development of French for specific purposes. This new branch of didactics aims to facilitate studies of non-native speakers at French or French speaking universities. Its concern is also to analyze the needs and difficulties of foreign students so that a tailored course enabling them to follow any course in their field of study could be worked up. The purpose of this article is to see if such an approach can be applied into a department of French where French language is at the same time aim, content and means of study. It will be briefly explained what French for academic purpose is all about. We will then outline the difficulties experienced by our students when writing their BA and MA theses. Finally we will take into account the practical aspects and difficulties of such an approach.

Keywords: French for specific purpose, French for academic purpose, academic writing, types of discourse, academic discourse, course design

L'idée d'intégrer une démarche issue du français sur objectif universitaire dans les programmes d'un département de français est le résultat de conversations informelles avec des enseignants-chercheurs qui constatent des difficultés d'ordre linguistique de plus en plus grandes dans les travaux de licence qu'ils ont à diriger. Ces enseignantschercheurs sont à distinguer des enseignants de langue dans le sens où ils sont en majorité en charge d'un enseignement disciplinaire, le plus souvent conduit en français. Dans ce cas, la langue française n'est donc pas un but en soi, au contraire des enseignements de PNJF, mais un vecteur d'enseignement d'une discipline. Or il s'avère que de plus en plus souvent, ces directeurs de mémoire doivent intervenir sur la qualité linguistique des productions des étudiants. Ce phénomène s'est amplifié ces dernières années du fait de la baisse de niveau des étudiants qui entrent en première année de français, d'autant plus que, dans un secteur de plus en plus concurrentiel, un certain 
nombre de départements de français dans les universités polonaises ont été contraints d'ouvrir leurs portes à des étudiants ayant un niveau débutant ou faux débutant. $\mathrm{Ce}$ nouveau public a demandé de gros efforts d'adaptation de notre offre d'enseignement. Ceci est d'autant plus problématique que quel que soit le niveau à l'entrée, un étudiant doit être en mesure, une fois en $3^{\text {ème }}$ année de rédiger un mémoire de licence en français. Au dire de certains enseignants, c'est loin d'être le cas.

Cette problématique évoque dans une certaine mesure celle rencontrée par les universités françaises confrontées à un afflux d'étudiants étrangers du fait d'une mobilité étudiante toujours croissante. Venus étudier les sciences, le droit, l'économie, la sociologie ces étudiants sont confrontés à certaines difficultés d'adaptation à un enseignement universitaire dans une langue qui n'est pas la leur. À ces difficultés linguistiques s'ajoutent les obstacles d'une "culture" universitaire souvent très différente de celle de leur pays d'origine.

Pour pallier les difficultés de ces étudiants allophones, importants pour le prestige d'une université à l'internationale, de nombreuses institutions d'enseignement supérieur ont mis en place des formations linguistiques afin de faciliter l'intégration de ces étudiants aux niveaux de langues très variables et aux profils universitaires de plus en plus divers mais avec un point commun, l'intégration à l'enseignement supérieur en langue française. Ces formations et donc la réflexion des enseignants en aval de celles-ci ont conduit à l'émergence d'un nouveau concept dans le paysage de la didactique du FLE: le français sur objectif universitaire, ou FOU.

Notre propos dans cet article est d'envisager la possibilité d'appliquer cette démarche didactique à un enseignement de français au sein d'un département de langues étrangères. Si les circonstances et le public sont différents il n'en reste pas moins que nos étudiants suivent un enseignement supérieur en langue française où la langue est à la fois objectif, contenu et vecteur d'enseignement. Après avoir rappelé brièvement en quoi consiste le français sur objectif universitaire, nous envisagerons la possibilité d'appliquer une telle démarche dans un département de langue française en soulignant brièvement les difficultés auxquelles doivent faire face nos étudiants. Pour finir, nous amorcerons une réflexion sur le traitement didactique à apporter aux problèmes et difficultés mis en évidence.

\section{LE FOU, UNE DÉCLINAISON DU FOS}

\subsection{DÉFINITION}

Mangiante et Parpette ont défini le FOU comme une « déclinaison du FOS, dans son approche centrée sur la connaissance la plus poussée des besoins d'un public ciblé, dans son parti pris de considérer que la réussite du projet d'intégration universitaire nécessite une maitrise linguistique autour de situations de communication spécifiques 
à la vie universitaire dans son ensemble » (Mangiante et Parpette, $2011: 5$ ). Les formations mises en place par les universités en France pour faciliter cette intégration, tournent le plus souvent autour de quatre axes : la langue, la méthodologie universitaire, le contexte culturel, les composantes institutionnelles. Si en France, ces quatre aspects sont importants pour l'intégration des étudiants étrangers, dans notre cas, seuls les deux premiers nous intéressent.

\subsubsection{EN QUOI CONSISTE UNE DÉMARCHE FOU?}

Celle-ci comprend les étapes suivantes : identification de la demande, analyse des besoins, collecte des données, analyse des données et enfin élaboration didactique. Dans le cas qui nous intéresse, il n'y a pas de demande claire mais plutôt un constat. Nos étudiants ont de plus en plus de difficulté à rédiger leur mémoire de licence. L'idée d'appliquer une démarche de FOU dans notre enseignement aurait donc pour but de remédier à ces difficultés. Encore faut-il clairement les identifier. D'autre part, outre ces difficultés de rédaction, qu'en est-il de la compréhension du discours scientifique, tant écrit que oral et donc des cours magistraux et des travaux dirigés conduits en français?

\section{ANALYSE DES BESOINS}

Cette première phase doit permettre d'identifier les situations de communications universitaires à maîtriser. Dans le contexte d'une philologie romane, on peut en voir deux, à savoir comprendre et restituer un cours et rédiger un mémoire de licence en $3^{\text {ème }}$ année et un mémoire de master en $5^{\text {ème }}$. Les deux grandes compétences mobilisées sont donc la compréhension orale et l'expression écrite. Outre la rédaction du mémoire, cette dernière est importante dans l'évaluation puisque celle-ci se fait essentiellement à l'écrit.

\subsection{COMPRENDRE UN COURS EN FRANÇAIS}

Dans un département de langue française, une bonne partie de l'enseignement est consacré à l'apprentissage de la langue française en tant que telle. À cet enseignement pratique s'ajoute un enseignement théorique, notamment en didactique, littérature, linguistique, traductologie. Ces cours sont tout d'abord assurés en polonais, du fait d'un nombre de plus en plus grand d'étudiants qui commencent des études de français avec un niveau débutant, ou faux débutant, donc dans l'incapacité de suivre des cours en français. Toutefois, dès la deuxième année, le polonais cède la place au français. Une 
enquête menée auprès des enseignants du département montre que dans leur grande majorité, ceux-ci utilisent rarement le français, même s'ils ont à faire à des « débutants $»^{1}$. Certains choisissent d'utiliser le polonais pour expliquer le déroulement et les objectifs du cours ainsi que les critères d'évaluations. Cette habitude disparaît presque complètement en troisième année. Les cours se font donc quasi exclusivement en français, de la deuxième à la dernière année.

Dans les cours qui supposent une interaction entre les enseignants et les étudiants, que nous appellerons travaux dirigés (TD), le français est là encore, la langue dominante, de la $2^{\text {ème }}$ à la $5^{\text {ème }}$ année. En $2^{\text {ème }}$ et $3^{\text {ème }}$ année le polonais peut être utilisé pour remédier à des difficultés d'expression. Notre enquête montre qu'il s'agit le plus souvent non pas d'une initiative des enseignants mais des étudiants. Il arrive que ceux-ci demandent en effet à l'enseignant de traduire ou d'expliquer en polonais un mot ou un concept théorique. De plus, des enseignants ont signalé l'habitude qu'ont certains étudiants de répondre en polonais à une question posée en français. La fatigue est une des raisons fréquemment invoquées par les étudiants. On argumentera bien évidemment que celle-ci masque en fait une insécurité linguistique tant du point de vue de la capacité à prendre la parole en public que d'un manque de lexique, de base, dirons-nous, mais aussi spécifique, lié à la discipline en question. Ce manque de vocabulaire est justement un des domaines qui intéresse le FOU puisque dans les universités françaises concernées par le problème de l'intégration des étudiants une partie des formations FOU mises en place traite du vocabulaire spécifique à la/aux discipline(s) concernée(s).

Cette habitude de demander des précisions ou de traduire des mots en polonais est confirmée par l'enquête que nous avons menée auprès des étudiants de $2^{\text {ème }}$ et $3^{\text {ème }}$ année. L'écrasante majorité indique demander de temps en temps à l'enseignant de fournir des explications en polonais. Ceci n'est pas surprenant dans la mesure où le contenu du cours et le manque de vocabulaire lié à la discipline sont indiqués comme étant des obstacles à la compréhension des cours par $90 \%$ des étudiants interrogés ${ }^{2}$. Ainsi, une première difficulté apparaît : l'appréhension du savoir dispensé pendant un cours. C'est d'autant plus intéressant que de nombreux enseignants dans les universités françaises constatent que les étudiants français ont également des difficultés de réception, de prise de notes, de compréhension d'ensemble des données. Il s'agirait donc plus d'un problème de compétence académique que d'un problème linguistique (Parpette, 2010).

Toutefois, si on peut facilement concevoir que les étudiants français et polonais aient les mêmes difficultés du point de vue académique et qu'ils ne sont pas/plus en mesure de suivre un cours magistral, on peut tout aussi facilement argumenter qu'à cette difficulté « académique », s'ajoute une difficulté plus « linguistique » pour nos étudiants qui suivent un cours dans une langue qui n'est pas la leur et que pour

${ }^{1}$ Ils ne le sont plus réellement puisqu'ils ont une année de français derrière eux. Mais nous utiliserons ce terme pour les différencier des étudiants qui sont entrés à l'université après avoir appris le français certains au lycée, d'autres depuis le collège.

${ }^{2}$ Quarante étudiants ont répondu à notre enquête. 
certains, ils n'avaient jamais étudiée avant de rentrer à l'université. Ainsi, les étudiants interrogés sur les difficultés liées à l'aspect linguistique d'un cours conduit en français ont répondu que ce qui leur posait le plus de problème c'était la compréhension orale, ce qui ne peut que provoquer des difficultés de réception du cours et dans la prise de note. Ils ont également indiqué avoir des difficultés à s'exprimer durant les TD³ .

Comme on pouvait s'y attendre, nos étudiants ont donc des difficultés à comprendre des cours majoritairement donnés en français. Ces difficultés sont d'ordres linguistiques et académiques. La question qui se pose alors est de faire la part entre les deux et de savoir quels liens elles entretiennent entre elles. Selon Pollet, la comparaison des difficultés des étudiants francophones et allophones montrent que « la méconnaissance de ces derniers du système linguistique est bien plus importante que celle des francophones et que celle-ci est l'obstacle premier (sans pour autant être le seul) à leur appropriation des discours universitaires » (Pollet, 2010 :138).

\subsection{AUTRE TYPE DE DISCOURS ET PREMIER ÉCRIT UNIVERSITAIRE : LE MÉMOIRE DE LICENCE}

Ce mémoire clos un premier cycle d'étude et s'il est moins ambitieux qu'un mémoire de maîtrise, il n'en reste pas moins important car il s'agit d'un premier travail de réflexion dans un domaine de spécialisation et, comme tout écrit universitaire, celui-ci obéit à des règles de composition et de rédaction précises. Par ailleurs, si comprendre et respecter la méthodologie de la rédaction d'un mémoire n'est pas forcément très simple pour des locuteurs natifs, elle l'est encore moins pour des étudiants qui ne maîtrisent pas la langue dans laquelle ils doivent rédiger ce travail.

Notre enquête auprès des enseignants montre que non seulement le niveau de langue des travaux est médiocre mais que très souvent, il est un obstacle à leur bonne compréhension. Au dire de ceux-ci, les fautes qui reviennent le plus souvent sont, sans surprise, des fautes de type morphosyntaxique avec des calques du polonais. À ces fautes de bases, s'ajoutent des fautes plus graves qui représentent de sérieux obstacles à la bonne compréhension des travaux. Il s'agit notamment des fautes de cohérence discursive en général et d'un manque de maîtrise des connecteurs logiques en particulier. Ceux-ci sont soit absents, soit mal utilisés, soit en excès. L'absence de connecteurs logiques n'est pas un problème en soi dans la mesure où il y a d'autres marqueurs grammaticaux et lexicaux qui contribuent à la cohérence d'un texte. La mauvaise utilisation ou l'excès de connecteurs logiques est en revanche plus problématique. Troisième type de fautes, les fautes lexicales avec notamment des fautes de registre. Les étudiants utilisent un registre de langue inadapté à ce type d'écrit, souvent "populaire" et ils ne maîtrisent qu'imparfaitement la terminologie nécessaire

\footnotetext{
${ }^{3}$ Là encore avec un chiffre proche de $90 \%$.
} 
à leur spécialité et inhérente à ce type de travail. Quelques enseignants ont enfin souligné des questions de styles et de phraséologie inadaptés à ce type d'écrit.

Là encore, une comparaison avec ce qui se passe dans les universités francophones peut apporter un éclairage intéressant. En effet, en comparant les travaux d'étudiants francophones et allophones on remarque que si les francophones n'ont pas ou peu de problèmes morphosyntaxiques, beaucoup de leur travaux se caractérisent par une absence totale de cohérence. Les francophones et les allophones semblent donc partager les difficultés relatives à « la situation d'énonciation, aux genres de discours et à leur organisation » (Pollet, $2010: 136)$. On en revient donc à la question du lien entre compétence académique et compétence linguistique. À cet égard, on peut toutefois penser que si un étudiant non francophone doit écrire en français et qu'il est dans l'incapacité à par exemple, argumenter ou justifier, c'est plutôt par manque de compétence linguistique liée à ce type d'énoncé (Cavalla, 2010).

Ainsi, si les problèmes de morphosyntaxe s'expliquent aisément par le fait qu'on écrit dans une langue étrangère, ceux liés à la cohérence discursive peuvent s'expliquer par un manque de compréhension des enjeux et des règles d'écriture d'un tel exercice mais également par un manque de compétences linguistiques permettant de respecter ces règles d'écriture. Outre l'aspect lexical précédemment évoqué, une démarche FOU s'intéresse également au développement des capacités linguistiques nécessaires à la rédaction des différents écrits universitaires.

\section{RÉFLEXION POUR LE TRAITEMENT DIDACTIQUE}

\subsection{L'ÉCRIT}

Les difficultés linguistiques brièvement décrites ci-dessus pourraient être analysées de deux points de vue : d'une part elles peuvent être envisagées comme la conséquence d'une compétence linguistique défaillante, mais d'autre part, elles peuvent être également les symptômes d'une « difficulté à entrer dans ce type de communication, d'une difficulté à construire du sens » (Pollet, 2010 : 139). Outre une aide linguistique, il faut donc apporter une aide méthodologique aux étudiants. En effet, rédiger un mémoire de licence ou de maitrise est un acte complexe et pour ce faire, ils doivent maîtriser quatre types de savoirs : scientifique, méthodologique, terminologique et linguistique (Cavalla, 2010). C'est donc sur ces quatre types de savoirs qu'il faut intervenir.

\subsubsection{L'ASPECT SCIENTIFIQUE ET TERMINOLOGIQUE}

Il s'agit ici pour l'étudiant d'acquérir un savoir dans un domaine de spécialité dans le cadre duquel il écrira son mémoire de licence et éventuellement son mémoire 
de maitrise. On peut raisonnablement assumer que cette acquisition se fait d'abord par les cours magistraux et travaux dirigés dont nous avons parlé plus haut. Or si on part du principe que ces cours sont assurés essentiellement en français et que les étudiants interrogés soulignent des difficultés de compréhension liées entre autre avec le contenu, on peut donc assumer que la réception du savoir pose problème. Faciliter la compréhension des cours nous semble donc un aspect important du travail d'accompagnement des étudiants dans la rédaction de leur mémoire. Nous y reviendrons un peu plus loin dans cet article.

\subsubsection{L’ASPECT MÉTHODOLOGIQUE ET LINGUISTIQUE}

Un écrit universitaire, quel qu'il soit, est un exercice d'écriture codé avec des exigences rédactionnelles strictes. Les comprendre et les maîtriser est donc indispensable et certains partent du principe que l'aspect méthodologique est indissociable de l'aspect linguistique : " la rédaction universitaire requiert des savoirs et des savoir-faire linguistiques «normés » et liés à la méthodologie » (Cavalla, $2010: 159)$. En analysant les caractéristiques discursive, pragmatique, lexicale et syntaxique d'un mémoire tel que doivent le rédiger nos étudiants nous pourrions vérifier cette hypothèse, dégager des tendances de rédaction et ainsi cerner les savoirs et savoir-faire à maîtriser.

En outre, certains écrits scientifiques se caractérisent par des tournures langagières, une phraséologie spécifique à ce genre d'écrits et à la discipline en question. Si c'est particulièrement vrai pour les sciences dites « dures » dont les écrits sont très codifiés, la question se pose pour les sciences humaines et, en ce qui nous concerne, pour les langues. Là encore, une analyse des écrits dans nos domaines permettraient le cas échéant de dégager des structures langagières récurrentes et une phraséologie plus ou moins normée.

Il pourrait être intéressant d'analyser dans un premier temps des productions réelles en y recherchant des énoncés considérés comme fautifs et d'en analyser les causes possibles de sorte à établir une sorte de « grammaire des fautes » (Pollet, 2010).

Enfin, on pourrait envisager d'établir un référentiel des savoirs et savoir-faire linguistiques nécessaires à la rédaction d'un mémoire.

\subsection{AMÉLIORER LA COMPRÉHENSION DES COURS}

Comme nous l'avons souligné plus haut, améliorer la compréhension des cours permettrait de développer les compétences scientifiques et terminologiques des étudiants. De plus, ces cours et les lectures conduites en annexes de ceux-ci peuvent également contribuer à améliorer les compétences méthodologiques et linguistiques, telles que brièvement esquissées auparavant. En effet, la lecture d'articles et d'ouvrages spécialisés permet de se familiariser avec les types de discours attendus lors de la rédaction d'un mémoire, avec le lexique spécialisé, avec une certaine phraséologie. 
Un cours universitaire est un événement complexe qui se caractérise par plusieurs niveaux de discours. C'est à la fois une présentation de certaines notions disciplinaires, le regard critique d'un enseignant par rapport à ces notions, un discours pédagogique et scientifique, un discours objectivé et impliqué (Mangiante et Parpette, 2011). Tout ceci amène à la question de savoir ce que signifie comprendre un cours et notamment l'essentiel de ce que cherche à transmettre l'enseignant. Cela ne peut se faire que si l'étudiant arrive à faire la part des choses entre les différents niveaux d'informations qui caractérisent cette production complexe. Pour Carette, l'étudiant doit être capable de « reconnaître le discours qu'il reçoit, son thème, identifier les changements de thèmes, identifier ce que fait l'enseignant (donne-t-il un exemple ? Une définition ? Fait-il un commentaire personnel ? etc.). Il y a donc un travail de familiarisation aux différents aspects du discours de l'enseignant » (Carette, 2010 : 99). Il faudrait donc analyser les caractéristiques de nos cours pour en dégager des éléments qui serviraient de base à un enseignement de familiarisation aux discours académiques oraux.

D'autre part, se repérer dans les différents niveaux d'information contenue dans un cours suppose là encore la maîtrise de certains savoirs et savoir-faire linguistiques de manière à pouvoir identifier, par exemple, le passage d'un thème à un autre sans que l'enseignant ait besoin de le signaler de manière explicite. Ce travail sur les compétences linguistiques nécessaires à la compréhension d'un cours aurait par ailleurs l'avantage de familiariser l'étudiant avec du lexique et des structures transposables à d'autres types de discours (évaluation, soutenance, rédaction du mémoire, etc.).

\section{CONTRAINTES ET DIFFICULTÉS DANS LA MISE EN PLACE D’UNE TELLE DÉMARCHE}

\subsection{LA COLLECTE DES DONNÉES}

Pour remédier aux difficultés de nos étudiants, il conviendrait donc analyser les discours qu'ils ont à comprendre et à produire pour en dégager les caractéristiques de manière à pouvoir travailler sur les compétences langagières et méthodologiques nécessaires à leur maîtrise. Tout ce travail correspond aux phases de collecte et d'analyse de données. Si la collecte de documents écrits est chose aisée, celle de document oraux, particulièrement de cours, est moins facile. Au delà des questions techniques, il faut avoir l'entière coopération des enseignants concernés.

\subsection{LA CONCEPTION ET L'ORGANISATION D'UN COURS DE FOU}

Cette première étape permettrait donc la création d'un référentiel de formation linguistique qui servirait de base à la construction d'un dispositif d'apprentissage. 
Mais on pourrait également envisager une réflexion commune entre enseignants de langue et enseignants-chercheurs afin d'examiner les attentes de ces derniers en termes de compétences langagières.

La question qui se pose ensuite est de savoir comment intégrer un cours spécifique de préparation aux discours universitaires dans un enseignement déjà bien chargé. Faut-il rajouter un cours ? Modifier le contenu des cours de FLE déjà existants ? On considérera qu'un cours à part sur la méthodologie de la rédaction d'un mémoire semble indispensable. Pour des difficultés autres que méthodologiques, on pourrait envisager de modifier le contenu de certains cours afin d'y inclure certaines compétences nécessaires. Par exemple, la question de la cohérence textuelle, fréquemment cité par les enseignants comme obstacle à la compréhension des travaux écrits peut facilement se travailler dans des cours déjà existants, puisqu'il s'agit d'une compétence qui n'est pas spécifique au discours académique.

Quant au dispositif d'aide à la compréhension des cours, pourquoi ne pas envisager des modules de formation sur une plateforme d'enseignement à distance telle que Moodle ? Celle-ci pourrait également servir de support à des activités pédagogiques spécifiques à chaque discipline.

\section{CONCLUSION}

Si on considère le FOU comme un outil d'aide à l'intégration dans un enseignement universitaire en français, alors cette démarche pourrait avoir toute sa place dans un département de langue française à l'étranger dans la mesure où tout ou partie de l'enseignement se fait en français. Une démarche FOU consiste en effet à concevoir un dispositif de formation qui remédie spécifiquement aux difficultés langagières des étudiants confrontés à des discours universitaires, oraux comme écrits. Cette démarche s'attache d'abord à l'analyse des besoins et des difficultés pour ensuite dégager des domaines d'intervention et proposer des formations adaptées. De manière plus spécifique nous avons vu qu'il faut intervenir sur la capacité des étudiants à rédiger un mémoire de licence et dans une moindre mesure un mémoire de maitrise. Mais on peut difficilement dissocier ce type d'écrit universitaire des cours qui le soustendent. Ainsi il nous semble important de s'intéresser également à la capacité des étudiants à comprendre un cours magistral ou des travaux dirigés conduit en français. Les capacités linguistiques nécessaires à cela sont certes abordées durant le cursus de français pratique mais peut-être de manière trop fractionnée et trop déconnectée des cours magistraux. Un cours de français orienté FOU pourait alors se concentrer sur l'application de ces savoirs et savoir-faire à la compréhension et à la production de discours académiques. Un tel cours pourrait également développer la maitrise du lexique propre à chaque discipline. Il faut enfin garder à l'esprit que méthodologie et langue peuvent difficilement être dissociés et que ces deux aspects doivent être 
travaillés simultanément. D'où l'importance là encore d'un cours spécifique puisque cette méthodologie peut difficilement être abordée, ou alors de manière parcellaire, lors d'un cours magistral dont le but premier est la transmission d'un savoir.

\section{RÉFÉRENCES}

CARETte, Emmanuelle (2010): «Aider les étudiants allophones à comprendre des cours universitaires : du parcours imposé vers le balisage pour randonnée en liberté ». Le français dans le monde/Recherches et applications 47: 94-105.

Cavalla, Christelle (2010): " Méthodologie d'apprentissage de l'écrit universitaire ». Le français dans le monde/Recherches et applications 47: 153-161.

MAngiante, Jean-Marc; PARPETte, Chantal (2011): Le français sur objectif universitaire. Grenoble : PUG.

PARPetTe, Chantal (2010): «Quelques réflexions sur des pratiques croisées entre formation linguistique et enseignement disciplinaire ». Le français dans le monde/Recherches et applications 47: 106-115.

Pollet, Marie-Christine (2010): «L'acculturation des étudiants aux discours universitaires : allophones, francophones, mêmes problèmes, mêmes combats ? ». Le français dans le mondel Recherches et applications 47: 133-141.

Goes, Jan; Mangiante Jean-Marc (2010): « Les écrits universitaires : besoins linguistiques et méthodologiques des étudiants allophones ». Le français dans le monde/Recherches et applications 47: 142-152. 